\title{
Detección precoz de infección por citomegalovirus en pacientes sometidos a trasplante alogéneico de precursores hematopoyéticos por reacción de polimerasa en cadena cuantitativa en tiempo real
}

\author{
M. Elena Ceballos, Cecilia Vizcaya, Daniela Pavez, Jaime Cerda, \\ Constanza Martínez-Valdebenito, Luisa Montecinos y Marcela Ferrés
}

\section{Early detection of cytomegalovirus infection in allogeneic hematopoietic stem cell transplant patients by real time-quantitative PCR}

Introduction: CMV pp65-antigenemia (antigenemia) has been used for monitoring CMV viremia in allogeneic hematopoietic stem cell transplant (aHSCT) recipients. Recently, real time quantitative PCR (RT-qPCR ) has been used as a better approach than antigenemia for CMV diagnosis. The objective of this study was to assess the correlation of CMV viremia between RT-qPCR and antigenemia in aHSCT patients. Material and Methods: Observational prospective study of all aHSCT patients during 10 months in our center. CMV RT-qPCR in whole blood was performed weekly from day +7 to +100 after aHSCT. Simultaneous antigenemia was performed from engrafment to day +100 . Concordance between both assays was evaluated. Results: Eighteen patients were included. In 120 simultaneous samples, 96 were concordant by both methods (80\%). Kappa coefficient was 0.583 . In $42 \%$ of cases without concordant results, patients were on antiviral therapy. Thirteen patients $(72 \%)$ developed CMV infection (20 episodes). In 17 episodes, both the antigenemia and CMV RT-qPCR were positive. CMV RT-qPCR was detectable 1-2 weeks before antigenemia in $45 \%$ of the episodes. Conclusion: Both methods had a moderate concordance and CMV RT-qPCR detects CMV reactivations earlier than antigenemia, especially in neutropenic patients.

Key words: Cytomegalovirus; quantitative real time PCR; antigenemia; haematopoietic stem cell transplantation; viremia.

Palabras clave: Citomegalovirus; reacción de polimerasa en cadena cuantitativa en tiempo real; antigenemia; trasplante de precursores hematopoiéticos, viremia.

\section{Introducción}

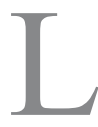

a infección por citomegalovirus (CMV) es una de las mayores causas de morbilidad y mortalidad en $/$ pacientes inmunocomprometidos, especialmente en trasplante de precursores hematopoyéticos alogénicos (TPHa) ${ }^{1}$.

Actualmente no existe consenso en cuál prueba de laboratorio debe ser utilizada como estándar de oro para el diagnóstico de infección por CMV antes del inicio de la enfermedad sintomática. Básicamente existen dos técnicas para monitorizar la ocurrencia de viremia en estos pacientes: la antigenemia pp65, que detecta a través de inmunofluorescencia una fosfoproteína de CMV en el núcleo de leucocitos neutrófilos, cuando el virus se encuentra en replicación activa $\mathrm{y}$, alternativamente, la reacción de polimerasa en cadena cuantitativa en tiempo real (en inglés RT-qPCR) que amplifica y cuantifica un gen de CMV en sangre total o plasma ${ }^{2}$.
La antigenemia se ha usado por varios años y hay experiencia en su uso e interpretación del significado clínico de sus resultados, pero requiere de leucocitos para un buen rendimiento del ensayo, condición que no siempre es posible en TPHa.

La RT-qPCR es más rápida y sensible, especialmente cuando la carga viral (CV) se realiza en sangre total comparada con plasma ${ }^{3-6}$.

En la práctica clínica, se han utilizado dos estrategias principales para prevenir la enfermedad por CMV. La primera es la profilaxis universal (se indica ganciclovir al receptor en riesgo de infección por CMV desde el prendimiento hasta el día +100 después del TPHa). La segunda estrategia, la más utilizada, es la terapia anticipada "preemptive therapy", donde sólo se indican antivirales cuando hay evidencias de laboratorio de replicación viral ${ }^{3}$, esto basado en la observación que la presencia de viremia aumenta el riesgo de enfermedad por $\mathrm{CMV}^{4}$.

Múltiples estudios que comparan antigenemia y
Pontificia Univeridad Católica de Chile, Santiago. Escuela de Medicina Departamento de Enfermedades Infecciosas del Adulto (MEC). División de Pediatría ((CV, DP, MF). Departamento de Salud Pública (JC) Laboratorio Infectología y Virología Molecular (CMV, LM).

Financiamiento: División de Pediatría y Laboratorio de Infectología y Virología Molecular de la Pontificia Universidad Católica de Chile.

Los autores declaran no poseer conflictos de interés.

Recibido: 13 de agosto de 2013 Aceptado: 11 de marzo de 2014

Correspondencia a: Marcela Ferrés Garrido mferres@med.puc.cl 
RT-qPCR para monitoreo de infección por CMV han mostrado que existe una correlación significativa entre ambos ensayos ${ }^{4,6-9}$. Un estudio de Sia y cols. ${ }^{10}$, mostró que hay una relación entre $\mathrm{CV}$ y desarrollo de enfermedad por $\mathrm{CMV}$, detectando viremia por ensayo molecular 14 días antes que la antigenemia.

Estos hallazgos son especialmente importantes en TPHa; diversas publicaciones muestran que los receptores de este tipo de trasplante pueden tener enfermedad por CMV sin antigenemia positiva ${ }^{11,12}$ durante períodos con y sin neutropenia. La técnica de RT-qPCR para CMV podría resolver este punto, pero se recomienda que los centros de trasplante evalúen las distintas técnicas disponibles y establezcan sus propios puntos de corte, antes de implementar un protocolo de cambio de una a otra técnica en pacientes sometidos a TPHa ${ }^{13}$.

El objetivo de este estudio fue evaluar la correlación de viremia por CMV a través de RT-qPCR con antigenemia, en pacientes que han recibido un TPHa.

Nuestra hipótesis es que existe una concordancia, al menos aceptable ${ }^{14}$, entre ambos ensayos de laboratorio y que la RT-qPCR permite una detección más temprana de la infección por CMV que la antigenemia, lo que facilitaría el inicio precoz de tratamiento y una menor frecuencia de enfermedad por CMV.

\section{Material y Métodos}

\section{Población estudiada}

Se incluyeron en forma consecutiva todos los pacientes que se sometieron a TPHa, con IgG positiva de CMV (ya fuese el donante y/o el receptor) en el Hospital Clínico de la Pontificia Universidad Católica de Chile (PUC), entre noviembre de 2010 y agosto de 2011. Se solicitó previamente consentimiento informado a los pacientes adultos y los tutores de pacientes pediátricos, en un formato aprobado por el Comité de Ética en Investigación de la Escuela de Medicina, PUC. Para niños sobre 7 años, se les solicitó además un asentimiento antes del inicio del estudio.

\section{Pruebas de laboratorio}

Se realizó RT-qPCR en sangre total semanalmente desde el día +7 después del trasplante hasta el prendimiento. Posteriormente se realizó en forma simultánea RT-qPCR y antigenemias en forma semanal hasta el día +100 después del transplante ${ }^{15}$. Ambas técnicas fueron realizadas en el Laboratorio de Infectología y Virología Molecular de la Escuela de Medicina de la PUC de Chile. La técnica de antigenemia se realizó de acuerdo a lo descrito por Landry M y cols. ${ }^{16}$, considerando un ensayo positivo la presencia de uno o más núcleos fluorescentes en 200.000 leucocitos. Se realizó el ensayo sólo en pacientes con recuento de leucocitos $\geq 1.000$ céls $/ \mathrm{ml}$ para conservar la confiabilidad de éste. Para la CV por reacción de polimerasa en cadena (RPC), se realizó la extracción por el equipo MagnaPure ${ }^{\circledR}$ ROCHE, siguiendo las instrucciones del fabricante según protocolo (ácido nucleico total o ADN). La amplificación se hizo en tiempo real con partidores y sondas incluidos en el ensayo de TibMol Biol- ROCHE $^{\circledR}$.

El umbral de detección de CMV fue de 200 copias/ $\mathrm{mL}$. El rango de detección lineal fue entre 500 copias $/ \mathrm{mL}$ y 20 millones de copias/mL y los valores obtenidos entre 200 y 500 copias $/ \mathrm{mL}$ se reportaron como positivos, pero sin un valor cuantificable.

Seguimiento clínico: los síntomas, signos, esquemas de profilaxis y terapias se obtuvieron de la ficha clínica.

\section{Definiciones $^{6}$}

- Reactivación por CMV: corresponde a la detección de viremia por CMV a través de antigenemia y/o RTqPCR en un paciente con o sin síntomas.

- Infección por CMV: fue la presencia de antigenemia positiva en un paciente asintomático.

- Síndrome CMV: se definió como una antigenemia positiva en un paciente con fiebre.

- Enfermedad por CMV: antigenemia y/o RT-qPCR positivos en un paciente con fiebre y compromiso de un órgano blanco.

- Prendimiento post TPHa: presencia de recuento absoluto de neutrófilos $\geq 500$ céls $/ \mathrm{ml}$ posterior al TPHa.

\section{Terapia}

Como estudio observacional, la terapia era indicada por el médico tratante utilizando los resultados de la antigenemia según criterio del médico clínico y del período posterior al trasplante en que se encontraba cada paciente. La RT-qPCR positiva no determinaba tratamiento anticipado, pero los resultados se reportaban al especialista. Sólo en los casos de enfermedad citomegálica, se trató a los pacientes que presentaban RT-qPCR positiva, aunque la antigenemia fuera negativa.

\section{Diseño}

Estudio prospectivo observacional.

\section{Análisis estadístico}

El número de pacientes estimados a reclutar fue de 16 , con un promedio de 10 muestras sanguíneas por cada uno. Dado que no existe un estándar de oro para el diagnóstico de infección por CMV, se evaluó la concordancia entre antigenemia y RT-qPCR mediante coeficiente kappa. Se utilizó la expresión cualitativa de la concordancia descrita por Cerda y cols ${ }^{14}$. Los parámetros demográficos fueron evaluados por estadísticas descriptivas. Se usaron porcentajes en las variables categóricas y desviación estándar en variables continuas. 


\section{Resultados}

Se incorporó al estudio un total de dieciocho pacientes con ТРHa, ingresando en forma consecutiva en un período de 10 meses. Cuatro de ellos fallecieron antes de completar el período de observación. Un paciente falleció por recaida de la leucemia y tres pacientes fallecieron cursando una neumonía. Sólo en uno de éstos se confirmó CMV como etiología y causa de muerte, el mes posterior al trasplante. Doce pacientes eran $\geq 18$ años. El promedio de edad fue de 22,7 años (rango 1-48 años). Doce pacientes eran hombres. Catorce pacientes $(78 \%)$ recibieron un injerto de donante no relacionado (11 de ellos de sangre de cordón umbilical). Dieciseis pacientes requirieron trasplante por enfermedades hematológicas malignas. Los 18 pacientes recibieron profilaxis antiviral con aciclovir. Las reactivaciones de CMV fueron tratadas con ganciclovir o valganciclovir. El diagnóstico basal de cada paciente y la fuente del injerto se presentan en la Tabla 1.

\section{Análisis por resultados de las pruebas de CMV}

De un total de 18 pacientes, se procesaron 186 muestras de sangre para antigenemia y 151 muestras para RT-qPCR.
Se obtuvo un promedio de 10,3 muestras de antigenemia y 8,4 muestras de RT-qPCR por paciente. Fueron 120 las muestras pareadas entre antigenemia y RT-qPCR. De esas 120 muestras simultáneas, 96 fueron concordantes entre ambos métodos $(80 \%)$ y 36 fueron positivas por ambas técnicas (Tabla 2). El coeficiente kappa fue de 0,583 [IC95\% 0,435-0,732] (fuerza de concordancia moderada).

La mayoría de las muestras positivas por RT-qPCR $(61 \%)$ estaban en rango no cuantificable (entre $200-<500$ copias $/ \mathrm{mL}$ ), por lo que no se pudo encontrar un punto de corte de CV para considerar inicio de terapia. No se encontró una correlación entre altos niveles de antigenemia y altos niveles de RT-qPCR (Tabla 3).

Se realizó el coeficiente de correlación de Pearson entre las variables $\log 10$ antigenemia y $\log 10$ carga viral con las 14 muestras que estaban sobre las 500 copias/ $\mathrm{mL}$ (correlación lineal) y tenían antigenemias positivas pareadas (Figura 1).

\section{Análisis de los resultados discrepantes}

Un total de 24 de 120 muestras (20\%) presentaron discrepancias entre antigenemia y RT-qPCR. Doce muestras tenían antigenemias positivas con RT-qPCR negativas.

\begin{tabular}{|c|c|c|c|c|c|c|c|c|c|}
\hline Paciente & Género & $\begin{array}{l}\text { Edad } \\
\text { (años) }\end{array}$ & Diagnóstico & $\begin{array}{c}\text { Tipo de } \\
\text { trasplante }\end{array}$ & $\begin{array}{l}\text { IgG citomegalovirus } \\
\text { antes del trasplante } \\
\text { Receptor/Donante }\end{array}$ & Remisión & $\begin{array}{c}\text { Prevención } \\
\text { enfermedad injerto } \\
\text { contra hospedero }\end{array}$ & $\begin{array}{l}\text { Antitimo- } \\
\text { globulina }\end{array}$ & $\begin{array}{c}\text { Reactivación de } \\
\text { citomegalovirus } \\
\text { sí/no }\end{array}$ \\
\hline 1 & Hombre & 21 & LLA calla+ & DR & $+/+$ & Sí & CSA/MTX & No & No \\
\hline 2 & Hombre & 1 & Wiscott-Aldrich & DNR & $+/+$ & No corresponde & CSA/MTX & Sí & Sí \\
\hline 3 & Mujer & 31 & LLA phi+ & $2 \mathrm{SCU}$ & $+/-$ & Sí & CSA/MMF & No & Sí \\
\hline 4 & Mujer & 16 & Mielodisplasia & $1 \mathrm{SCU}$ & $+/-$ & No corresponde & CSA/MMF & No & Sí \\
\hline 5 & Hombre & 24 & LMA & DR & $+/+$ & Sí & CSA/MTX & No & No \\
\hline 6 & Mujer & 22 & LMC & DR & $-1+$ & No & CSA/MTX & No & No \\
\hline 7 & Hombre & 38 & $\mathrm{LLC}$ & DNR & $+/+$ & Sí & CSA/MTX & Sí & Sí \\
\hline 8 & Hombre & 9 & LLA & $1 \mathrm{SCU}$ & $+/-$ & Sí & CSA/MMF & Sí & No \\
\hline 9 & Hombre & 17 & LMA & $2 \mathrm{SCU}$ & $+/-$ & Sí & CSA/MMF & No & Sí \\
\hline 10 & Hombre & 5 & $\begin{array}{c}\text { Anemia Blackfan } \\
\text { Diamond }\end{array}$ & $1 \mathrm{SCU}$ & $+1-$ & No corresponde & CSA/MMF & Sí & Sí \\
\hline 11 & Mujer & 20 & LMA & DR & $+/+$ & Sí & CSA/MTX & No & Sí \\
\hline 12 & Hombre & 41 & LLA phi+ & $2 \mathrm{SCU}$ & $+/-$ & Sí & CSA/MMF & No & Sí \\
\hline 13 & Hombre & 34 & Linfoma cutáneo T & DNR & $+/+$ & No & CSA/MTX & No & Sí \\
\hline 14 & Hombre & 48 & Mielodisplasia & $2 \mathrm{SCU}$ & $+/-$ & No corresponde & CSA/MMF & Sí & Sí \\
\hline 15 & Hombre & 18 & LLA & $1 \mathrm{SCU}$ & $+/-$ & Sí & CSA/MMF & No & Sí \\
\hline 16 & Mujer & 26 & LLA & $1 \mathrm{SCU}$ & $+/-$ & Sí & TACRO/MMF & No & Sí \\
\hline 17 & Mujer & 14 & LLA & $1 \mathrm{SCU}$ & $+/-$ & Sí & CSA/MMF & No & No \\
\hline 18 & Hombre & 24 & LLA & $2 \mathrm{SCU}$ & $+/-$ & Sí & CSA/MMF & No & Sí \\
\hline
\end{tabular}


Tabla 2. Muestras para detección de CMV por antigenemia y reacción de polimerasa en cadena

\begin{tabular}{lccc} 
Antigenemia & \multicolumn{2}{c}{ Carga viral por RT- qPCR } \\
Positiva & Negativa & Total \\
Positiva, $\mathrm{n}(\%)$ & $36(30)$ & $12(10)$ & 48 \\
Negativa, $\mathrm{n}(\%)$ & $12(10)$ & $60(50)$ & 72 \\
Total & 48 & 72 & $120(100)$
\end{tabular}

RT- qPCR: RPC en tiempo real.

Tabla 3. Correlación entre diferentes niveles de antigenemia y carga viral de CMV obtenidas de muestras positivas concomitantes

$\begin{array}{lccc}\text { Niveles de antigenemia } & \begin{array}{c}\text { Carga viral de CMV } \\ \mathbf{2 0 0 -} \begin{array}{c}\mathbf{5 0 0} \text { copias/ml } \mathbf{m l} \\ (\mathbf{n}=\mathbf{2 2})\end{array}\end{array} & \begin{array}{c}\text { Carga viral de CMV } \\ \mathbf{5} \mathbf{5 0 0} \text { copias/ml } \\ (\mathbf{n}=\mathbf{1 4})\end{array} & \text { Total } \\ \text { 1-5 céls positivas/200.000 leucocitos } & 17 & 4 & 21 \\ \geq 6 \text { céls positivas/200.000 leucocitos } & 5 & 10 & 15 \\ \text { Total } & 22 & 14 & 36\end{array}$

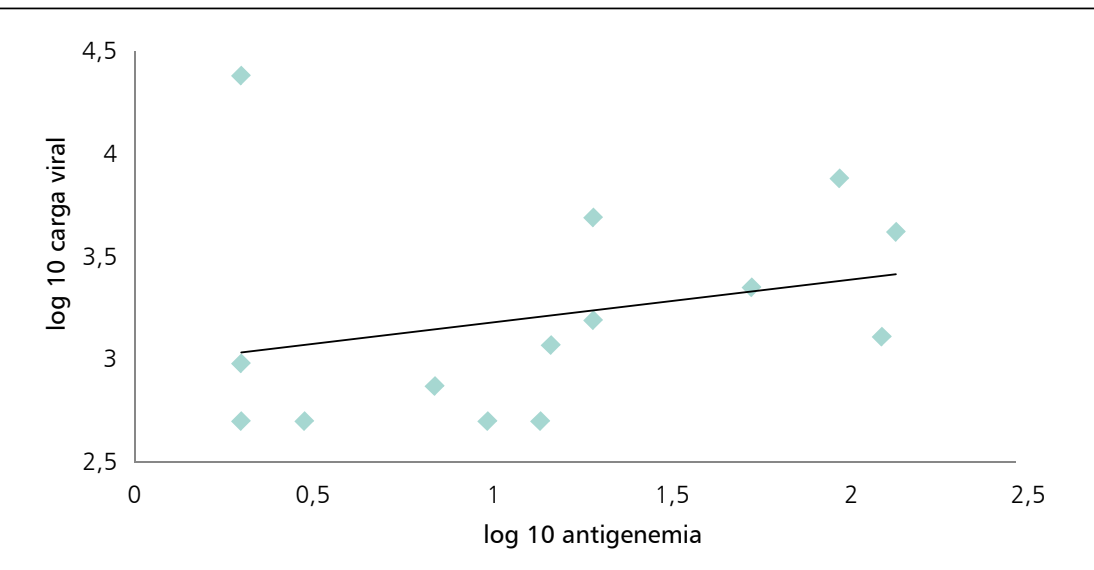

Figura 1. Concordancia entre antigenemia y carga viral de CMV por reacción de polimerasa en cadena en tiempo real. Coeficiente de correlación de Pearson. Concordancia entre $\log 10$ antigenemia CMV y log10 carga viral de CMV por RT-qPCR con las 14 muestras que se encontraban sobre 500 copias $/ \mathrm{mL}$ y tenían antigenemias positivas concomitantes. $(r=+0,267, p=0,357)$. Nota: ambas variables poseen una distribución normal. caso que presentó reactivación por CMV diagnosticada por antigenemia y no por RT-qPCR. Esto ocurrió en el día +82 post trasplante, el paciente estuvo asintomático y tenía bajo nivel de antigenemia ( tres células positivas en 200.000 leucocitos).

Doce casos presentaron RT-qPCR positivas con antigenemias negativas. Seis casos ocurrieron en pacientes que estaban en terapia antiviral al momento de la muestra. Dos casos fueron interpretados como RT-qPCR falsamente positivas (estos pacientes no tenían síntomas, sus antigenemias fueron siempre negativas y no necesitaron tratamiento antiviral hasta el día +100). Las CVs fueron 4.800 y 9.200 copias $/ \mathrm{mL}$ respectivamente y las CVs subsecuentes negativas. En cuatro casos las antigenemias se interpretaron como falsamente negativas por asociarse a manifestaciones clínicas compatibles con síndrome o enfermedad por CMV. En tres de estos pacientes la RT-qPCR fue positiva una a dos semanas antes de la antigenemia y en uno de ellos la RT-qPCR fue positiva en sangre y lavado bronco-alveolar, asociado a falla respiratoria, pero con antigenemia negativa. Este caso fue interpretado y tratado como enfermedad pulmonar por CMV y fue uno de los pacientes que falleció antes del día +100 .

En suma, se observaron 10 casos de resultados discordantes que estaban recibiendo ganciclovir (42\% de todas las discordancias), tanto en el grupo de antigenemia positiva- RT-qPCR negativa como en el grupo RT-qPCR positiva-antigenemia negativa.

\section{Análisis de los episodios de infección}

Trece pacientes (72\%) tuvieron 20 episodios de infección por $\mathrm{CMV}$, todos diagnosticados por antigenemia y tratados con ganciclovir. Diecisiete de los 20 episodios tenían antigenemia y RT-qPCR positivas concomitantes. De éstas, 14 fueron pacientes asintomáticos, uno tuvo síntomas sistémicos (fiebre) y dos presentaron enfermedad por CMV. De estos dos casos, uno tuvo diarrea prolongada al día +70 después del trasplante, con una biopsia compatible con colitis por CMV ("inclusiones citoplasmáticas" y RPC de CMV positiva en tejido) y el otro paciente tuvo neumonia y RPC CMV positiva en lavado bronco-alveolar y sangre al día +50 después del trasplante. Ambos presentaron RT-qPCR positiva en sangre una semana antes que la antigenemia (ambas con $<500$ copias $/ \mathrm{mL}$ ).

Los tres episodios no concordantes fueron: un paciente/ un episodio con antigenemia negativa, pero RT-qPCR positiva (con $<500$ copias $/ \mathrm{mL}$ ) en sangre y lavado bronco-alveolar. Los otros dos episodios ocurrieron en un paciente que siempre fue asintomático, tuvo antigenemias bajas (dos y tres células positivas por 200.000 leucocitos) y RT-qPCR negativas.

La RT-qPCR de CMV fue positiva una o dos semanas antes que la antigenemia en $45 \%$ de los episodios. 

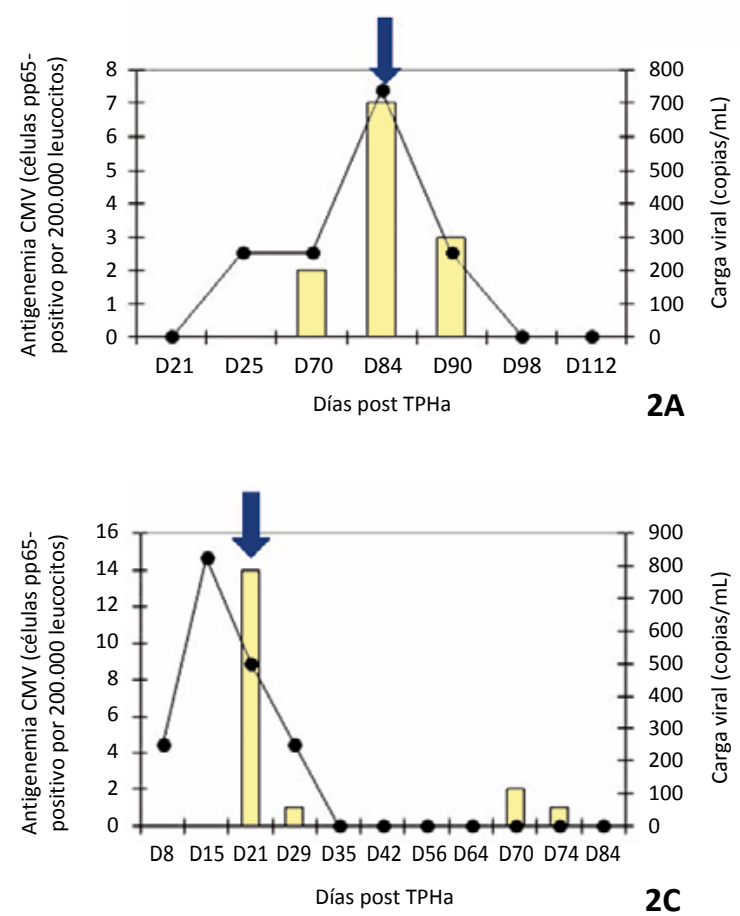

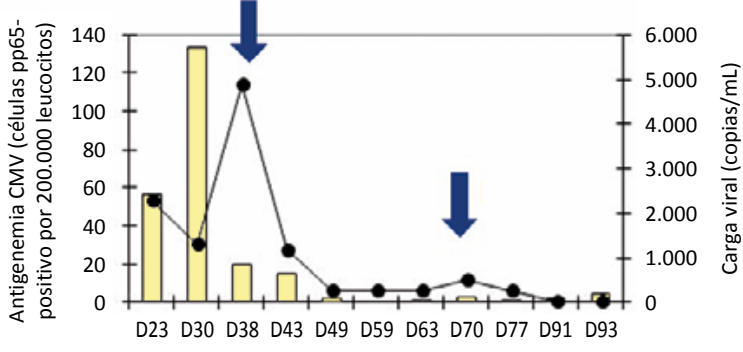

2B

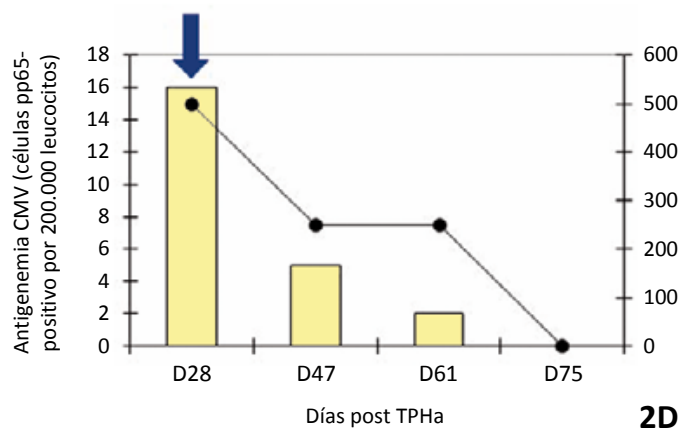

Figura 2. Curso clínico de reactivación de CMV en trasplantados de precursores hematopoieticos alogénicos. Muestras secuenciales de pacientes analizadas por carga viral de CMV por reacción de polimerasa en cadena en tiempo real (RT-qPCR) y antigenemia. Barras blancas: antigenemia (en núcleos/200.000 leucocitos). Puntos negros: carga viral CMV (copias/mL). Flechas: inicio de terapia antiviral. 2A (paciente número 4): concordancia entre RT-qPCR y antigenemia. El ensayo molecular fue positivo dos semanas antes que la antigenemia. 2B (paciente número 7): concordancia entre RT-qPCR y antigenemia. Durante la terapia con ganciclovir, la RT-qPCR se hizo negativa antes que la antigenemia. 2C (paciente número 9): concordancia entre RT-qPCR y antigenemia. El ensayo molecular fue positivo dos semanas antes que la antigenemia Las antigenemias positivas el día +70 y +74 fueron consideradas falsas positivas debido a que el paciente estaba asintomático, no fue tratado, presentando una evolución favorable. 2D (paciente número 10): Concordancia entre ambos ensayos.

De los 18 pacientes reclutados, seis presentaron enfermedad de injerto contra hospedero (cutánea, hepática e intestinal). De ellos, dos hicieron infección por CMV y uno presentó una posible neumonía por Aspergillus sp.

La Figura 2 muestra los valores de RT-qPCR y antigenemia de CMV en cuatro pacientes del estudio.

\section{Conclusión}

La infección por CMV es frecuentemente responsable de morbilidad y mortalidad en pacientes sometidos a TPHa. En nuestra cohorte, $72 \%$ de los pacientes presentaron infección por CMV, similar a lo descrito en otras publicaciones s $^{17,18}$ y en $15 \%$ se manifestó como enfermedad por CMV. Este alto porcentaje de reactivaciones se debe a que en nuestro centro, $60 \%$ de los pacientes tienen un alto riesgo de reactivación por CMV $(60 \%$ de los injertos fueron de sangre de cordón umbilical) y en $40 \%$ el riesgo es moderado (donante y/o receptor positivo). Algunos pacientes también hicieron otras complicaciones como enfermedad de injerto contra hospedero o neumonía fúngica, empeorando su pronóstico.

Durante años, la antigenemia pp65 ha sido utilizada para la detección de infección por CMV en nuestra institución. Sin embargo, este recurso no puede ser utilizado como examen de referencia diagnóstica; siendo un examen que se realiza con observación de leucocitos polimorfonucleares, no permite la monitorización de replicación de CMV antes del prendimiento medular, la que retrasa el diagnóstico en pacientes con granulocitopenias prolongadas. Otras desventajas son que su procesamiento es laborioso y que requiere un tecnólogo experimentado para su lectura e interpretación ${ }^{4,7,16,19}$. La necesidad de un procesamiento inmediato, hace difícil su almacenamiento y transporte desde lugares remotos, donde la prueba no está disponible ${ }^{8,16}$. Múltiples estudios han mostrado la utilidad de detectar infección por CMV a través de RT-qPCR. Este ensayo, comparado con la antigenemia, tiene varias ventajas; puede ser realizado en pacientes neutropénicos ${ }^{4,6,7,15,19}$, es más rápido de procesar 
y la estabilidad de la muestra favorece el almacenamiento y transporte desde y hacia otros hospitales.

En el presente estudio, se evaluó la correlación de viremia por CMV a través de RT-qPCR con antigenemia, en pacientes sometidos a TPHa. Debido a que la sensibilidad y especificidad de la RT-qPCR de CMV no puede ser estimada -en ausencia de un estándar de oro para el diagnóstico de esta infección-, se utilizó el coeficiente kappa para evaluar la concordancia con la antigenemia. Nuestro estudio obtuvo una concordancia kappa de 0,583 entre RT-qPCR y antigenemia (moderada fuerza de concordancia), superando lo planteado en nuestra primera hipótesis y a lo descrito en otras publicaciones ${ }^{17,20}$. La RT-qPCR fue positiva en algunos casos en que la antigenemia fue negativa o en que ésta no podía realizarse (pacientes intensamente neutropénicos). Estos hallazgos muestran que la RT-qPCR puede ser especialmente útil en las primeras semanas después del TPHa, cuando aún no ha ocurrido el prendimiento medular. Utilizando los resultados de la RT-qPCR, 45\% de los episodos de infección podrían haberse diagnosticado y tratado una a dos semanas antes de la positivizacion de la antigenemia. Este hallazgo confirma nuestra segunda hipótesis que la RT-qPCR permite una detección más temprana de la infección por CMV en comparación con la antigenemia, hallazgo también descrito en otras publicaciones ${ }^{10,20,21}$.

En este estudio, una importante proporción de las discordancias entre ambos exámenes (10/24; 42\%) ocurrieron durante el uso de terapia antiviral. Como Choi y cols. ${ }^{7}$ describieron, la exposición a terapia antiviral es un factor que influye significativamente en los resultados discrepantes. En estos casos, la RT-qPCR de CMV y la antigenemia parecen disminuir con distintas velocidades.

No pudimos determinar valores de corte de la RT-qPCR para inicio de tratamiento, debido a que las cargas virales encontradas estaban bajo el umbral lineal de detección. No obstante, los hallazgos sugieren que cualquier carga viral positiva ( $\geq 200$ copias $/ \mathrm{ml}$ ), independiente de su valor cuantificado, representa infección y debe ser tratada en estos pacientes.

En conclusión, la concordancia entre las dos técnicas y la detección más precoz de viremia con CV comparada con antigenemia, valida la utilidad de la RT-qPCR en TPHa como un mejor ensayo en el diagnóstico y manejo precoz de infección por CMV en estos pacientes.

Es importante destacar la falta de estandarización de los resultados de la CV y de la antigenemia con respecto a un estándar internacional al momento de realizar este estudio, lo cual impidió comparar los resultados obtenidos con los de otros centros. En los últimos años, los esfuerzos han permitido la aparición de un estándar de la Organización Mundial de la Salud para la CV de CMV que ha permitido la incorporación al mercado de exámenes IVD ("in vitro diagnostic products") que entregan resultados en unidades internacionale ${ }^{22}$, lo que conducirá a la realización de estudios prospectivos, colaborativos entre centros, de manera de obtener el seguimiento de un mayor número de pacientes y de esta manera establecer puntos de corte de la CV que indiquen la necesidad de inicio de terapia.

Agradecimientos. A María Wiestruck, Marcela Contreras, Bruno Nervi y Verónica Jara por incorporar sus pacientes al estudio.

\section{Resumen}

Introducción: La antigenemia pp65 (antigenemia) ha sido utilizada para monitorizar viremia de citomegalovirus (CMV) en pacientes sometidos a trasplantes alogeneicos de precursores hematopoiéticos (TPHa). Recientemente, la reacción de polimerasa en cadena cuantitativa en tiempo real (en inglés RT-qPCR) se ha usado como una mejor aproximación al diagnóstico de infección por CMV. El objetivo de este estudio fue evaluar la correlación de viremia por CMV a través de RT-qPCR con antigenemia, en pacientes que han recibido TPHa. Material y Métodos: Estudio prospectivo, observacional, de los pacientes sometidos a TPHa durante 10 meses. Se realizó RT-qPCR de CMV en sangre total semanalmente desde el día +7 al+100 después del trasplante y antigenemia en forma simultánea desde el prendimiento hasta el día +100 . Se evaluó la concordancia entre ambos ensayos. Resultados: Dieciocho pacientes fueron incluidos. En 120 muestras simultáneas, 96 fueron concordantes por ambos métodos (80\%). El coeficiente Kappa fue 0,583. En los casos no concordantes, el $42 \%$ se encontraba en terapia antiviral. Trece pacientes (72\%) desarrollaron infección por CMV (20 episodios). En 17 episodios, ambos ensayos fueron positivos. La carga viral fue detectable 1-2 semanas antes que la antigenemia en $45 \%$ de los episodios. Conclusión: Existe una buena correlación entre ambas técnicas y la RT-qPCR detecta más precozmente que la antigenemia las reactivaciones de $\mathrm{CMV}$, especialmente en pacientes neutropénicos. 


\section{Referencias bibliográficas}

1.- Ljungman P, Brand R, Einsele H, Frassoni F, Niederwieser D, Cordonnier C. Donor CMV serologic status and outcome of CMVseropositive recipients after unrelated donor stem cell transplantation: an EBMT megafile analysis. Blood 2003; 102 (13): 4255-60.

2.- Preiksaitisa J, Brennanb D, Fishman J, Allen U. Canadian Society of Transplantation Consensus Workshop on Cytomegalovirus Management in Solid Organ Transplantation Final report. Am J Transplant 2005; 5: 218-27.

3.- Habib K, Lamia T, Amel L, Abdelrahmen A, Saloua L, Hana E, et al. Time of onset, viral load, relapse, and duration of active cytomegalovirus infection in bone marrow transplant outcomes. Exp Clin transplant. 2008; 6: 67-73.

4.- Deback C, Fillet A, Dhedin N, Barrou B, Varnous S, Najioullah F, et al. Monitoring of human cytomegalovirus infection in inmunosuppressed patients using real-time PCR on whole blood. J Clin Virol 2007; 40: 173-9.

5.- Koidl C, Bozic M, Marth E, Kessler H. Detection of CMV DNA: Is EDTA whole blood superior to EDTA plasma?. J Virol Methods 2008; 154: 210-12.

6.- Ghaffari S, Obeidi N, Dehghan M, Alimoghaddam K, Gharehbaghian A, Ghavamzadeh A. Monitoring of cytomegalovirus reactivation in bone marrow transplant recipients by real-time PCR. Pathol Oncol Res. 2008; 14: 399-409.

7.- Choi S, Lee D, Lim J, Park S, Choi J, Yoo J, et al. Comparison of quantitative cytomegalovirus real-time PCR in whole blood and pp65 Ag CMV assay: clinical utility of CMV real-time PCR in hematopoietic stem cell transplant recipients. J Korean Med Sci 2009;
24: $571-8$.

8.- Li H, Dummer S, Estes W, Meng S, Wrigth P, Tang Y. Measurement of human cytomegalovirus loads by quantitative real-time PCR for monitoring clinical intervention in transplant recipients. J Clin Microbiol 2003; 41: 187-91.

9.- Allice T, Enrietto M, Pittaluga F, Varetto S, Franchello A, Marchiaro G, et al. Quantitation of cytomegalovirus DNA by real-time polymerase chain reaction in peripheral blood specimens of patients with solid organ transplants: comparison with end-point PCR and pp65 antigen test. J Med Virol 2006; 78: 915-22.

10.- Sia I, Wilson J, Groettum C, Espy M, Smith T, Paya C. Cytomegalovirus (CMV) DNA load predicts relapsing CMV infection after solid organ transplantation. J Infect Dis 2000; 181: 717-20.

11.- Ljungman P, Hakki M, Boeckh M. Cytomegalovirus in hematopoietic stem cell transplant recipients. Hematol Oncol Clin N Am. 2011; 25: 151-69.

12.- Gentile G, Picardi A, Capobianchi A, Spagnoli A, Cudillo L, Dentamaro T, et al. A prospective study comparing quantitative cytomegalovirus (CMV) polymerase chain reaction in plasma and pp65 antigenemia assay in monitoring patients after allogeneic stem cell transplantation. BMC Infect Dis 2006; 6: 167.

13.- Pang X L, Fox J D, Fenton J M, Miller G G, Caliendo A M, Preiksaitis J K, et al. Interlaboratory comparison of cytomegalovirus viral load assays. Am J Transplant 2009; 9: 258-68.

14.- Cerda J, Villarroel L. Evaluación de la concordancia inter-observador en investigación pediátrica: Coeficiente de Kappa. Rev Chil Pediatr 2008; 79 (1): 54-8.

15.- Tomblyn M, Chiller T, Einsele H, Gress R, Sepkowitz K, Storek J, et al. Guidelines for preventing infectious complications among hematopoietic cell transplantation recipients: A global perspective. Biol Blood Marrow Transplant 2009; 15: 1143-238.

16.- Landry M L, Ferguson D, Cohen S, Huber K, Wetherill P. Effect of delayed specimen processing on cytomegalovirus antigenemia test results. J Clin Microbiol. 1995; 33: 257-9.

17.- Li Y, Gao L, Wang L L, Ding Y, Xu Y Y, Li H H, et al. Surveillance of CMV infection in allo-HSCT recipients and guidance on preemptive therapy by RQ-PCR. Zhongguo Shi Yan Xue Ye Xue Za Zhi 2013; 21 (1): 161-8.

18.- Qiu Z X, Wang M J, Wang L H, Sun Y H, Xu W L, Liu W, et al. Quantitative monitoring of blood cytomegalovirus after allogeneic hematopoietic stem cell transplantation and its clinical significance. Zhonghua Nei Ke Za Zhi 2012; 51 (5): 371-5.

19.- Zaia J. Prevention of cytomegalovirus disease in hematopoietic stem cell transplantation. Clin Infect Dis 2002; 35: 999-1004.

20.- Farfán M, Torres J, Vergara A, Donoso G, Alba A, Paris C, et al. Comparison of real-time polymerase chain reaction and antigenemia assay to detect cytomegalovirus in pediatric transplants. Rev Chilena Infectol 2011; 28 (2): 113-7.

21.- Griscelli F, Barrois M, Chauvin S, Lastere S, Bellet D, Bourhis J H. Quantification of human cytomegalovirus DNA in bone marrow transplant recipients by real-time PCR. J Clin Microbiol 2001; 39 (12): 4362-9.

22.- Razonable R R, Åsberg A, Rollag H, Duncan J, Boisvert D, Yao J D, et al. Virologic suppression measured by a cytomegalovirus (CMV) DNA test calibrated to the World Health Organization international standard is predictive of CMV disease resolution in transplant recipients. Clin Infect Dis 2013; 56 (11): 1546-53. 